Claremont Colleges

Scholarship@ Claremont

All HMC Faculty Publications and Research

HMC Faculty Scholarship

$1-1-1987$

\title{
Wavenumber Selection of Convection Rolls in a Box
}

Wayne Arter

University of Arizona

Andrew J. Bernoff

Harvey Mudd College

A. C. Newell

University of Arizona

\section{Recommended Citation}

Wavenumber selection of convection rolls in a box. Wayne Arter, A. Bernoff, and A. C. Newell, Phys. Fluids 30, 3840 (1987).

This Article is brought to you for free and open access by the HMC Faculty Scholarship at Scholarship @ Claremont. It has been accepted for inclusion in All HMC Faculty Publications and Research by an authorized administrator of Scholarship @ Claremont. For more information, please contact scholarship@cuc.claremont.edu. 


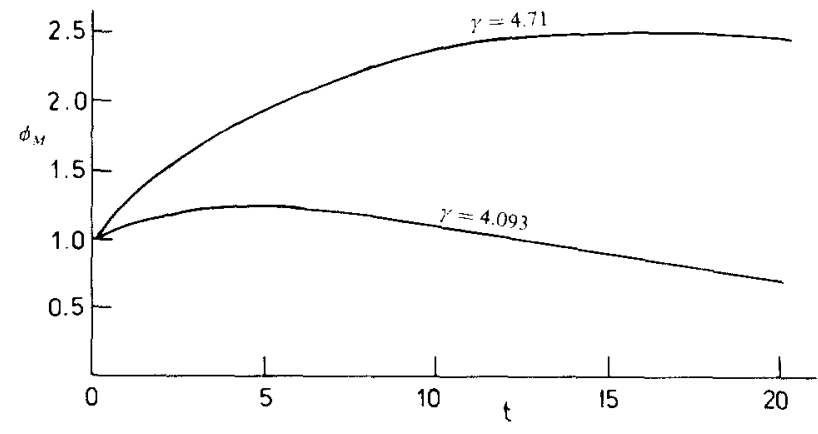

FIG. 4. Maximum of $|\phi|$ with respect to $\xi$ for $R=70, \gamma=4.71$ $(\alpha=0.0673$ ) and for $R=70, \gamma=4.093(\alpha=0.0584)$.

Many other cases were calculated with the following general results. For $\alpha$ less than about 0.2 , so that $R_{\text {crit }}(\alpha)<R_{2}(\alpha)$ we found the following.

(i) If $R<R_{\text {crit }}$ the wave packet decays steadily and rapidly as it moves downstream.

(ii) If $R>R_{\text {crit }}$ by a moderate amount up to about $10 \%$ of $R_{\text {crit }}$ the "packet" shows a slight growth before decaying downstream. However, we did not calculate any cases with $R$ more than marginally beyond $\mathscr{B}_{2}$. We also mention that Allmen, ${ }^{8}$ by a numerical solution of the time-dependent partial differential equation governing the evolution of a disturbance initially close to $\delta(\xi)$, obtained very similar results.
The above results may throw some doubt on the description of channel flows as "unstable" when $R>R_{\text {crit }}(\alpha)$. At the same time the results support the idea of "local instabilities" of Ref. 1, for although our calculations are for flow in a wedge, similar modes exist in curved-wall channels. ${ }^{6}$ For a curved-wall channel, where $\alpha(\xi)$ only locally exceeds the value on the stability boundary $R=R_{\text {crit }}(\alpha)$, it seems probable that isolated disturbances would grow slightly and then decay rapidly downstream. Thus Fraenkel's ${ }^{3}$ theory, including cases of separation and reattachment of the main stream, using values of $\alpha$ slightly beyond the boundary $\mathscr{B}_{2}$, may be in some cases a good approximation to the physics, albeit possibly modified by small oscillations near separation or reattachment in accord with the observations of Cherdron $e t$ al. ${ }^{9}$

${ }^{1}$ I. J. Sobey and P. G. Drazin, J. Fluid Mech. 171, 263 (1986).

${ }^{2}$ L. E. Fraenkel, Proc. R. Soc. London Ser. A 267, 119 (1962).

${ }^{3}$ L. E. Fraenkel, Proc. R. Soc. London Ser. A 272, 406 (1963).

${ }^{4}$ W. H. H. Banks, P. G. Drazin, and M. B. Zaturska, J. Fluid Mech., in press.

${ }^{5}$ P. M. Eagles and M. A. Weissman, J. Fluid Mech. 69, 241 (1975).

${ }^{6}$ G. A. Georgiou and P. M. Eagles, J. Fluid Mech. 159, 259 (1985).

${ }^{7}$ G. A. Georgiou, Ph.D. thesis, The City University, London E. C. 1, 1982.

${ }^{8}$ M. J. Allmen, Ph.D. thesis, The City University, London E. C. 1, 1980.

${ }^{9}$ W. Cherdron, F. Durst, and J. H. Whitelaw, J. Fluid Mech. 84, 13 (1978).

\section{Wavenumber selection of convection rolls in a box}

Wayne Arter, ${ }^{\text {a) }}$ A. Bernoff, and A. C. Newell

Department of Mathematics, University of Arizona, Tucson, Arizona 85721

(Received 15 June 1987; accepted 18 August 1987)

The dynamics of two-dimensional Rayleigh-Bénard convection rolls are studied in a finite layer with no-slip, fixed temperature upper and lower boundaries and no-slip insulating side walls. The dominant mechanism controlling the number of rolls seen in the layer is an instability concentrated near the side walls. This mechanism significantly narrows the band of stable wavenumbers although it can take a time comparable to the long (horizontal) diffusion time scale to operate.

Wavenumber selection mechanisms in Rayleigh-Bénard convection have been intensively studied, both because of their intrinsic interest, ${ }^{1}$ and also to help explain the transition to turbulence, particularly in convecting layers of large horizontal extent (shallow tanks). ${ }^{2}$ To proceed analytically, it has always been necessary to impose periodic or reflecting boundary conditions in either the horizontal ${ }^{2}$ or vertical ${ }^{3,4}$ direction. The more physically relevant problem of determining what roll wavenumbers are allowed in a box where all walls are no-slip has not previously been studied. Catton ${ }^{5}$ has performed the only work with experimentally realistic velocity boundary conditions that provides some insight into which wavenumbers are preferred in a box of large but finite, horizontal extent. However, Catton's work was linear, concerned with finding the smallest Rayleigh number $\mathrm{Ra}_{c}$ at which convection could occur and the corresponding flow field. Linear theory at best only tells which roll patterns will not decay back to the purely conducting, no-flow state: nonlinear effects can prevent many more patterns from appearing, or lead to their eventual destruction. This is the problem of wavenumber selection that our work addresses.

The equations for Rayleigh-Bénard convection are tak- 
en in dimensionless form:

$$
\frac{\partial \mathbf{u}}{\partial t}+\mathbf{u} \cdot \nabla \mathbf{u}=\sigma\left[-\nabla p+\nabla^{2} \mathbf{u}+\operatorname{Ra}(T-1+z) \hat{\mathbf{z}}\right],
$$

$\frac{\partial T}{\partial t}+\mathbf{u} \cdot \nabla T=\nabla^{2} T, \quad \nabla \cdot \mathbf{u}=0$.

Here, $\mathbf{u}, T$, and $p$ are the velocity, temperature, and pressure fields, respectively, made dimensionless with respect to depth $d$ and vertical diffusion time $d^{2} / \kappa$, where $\kappa$ is the thermal diffusivity. The Prandtl number $\sigma=v / \kappa$, where $v$ is viscosity, was fixed at $\sigma=2.5$ (typical of water at $70^{\circ} \mathrm{C}$ ). Gravity acts in the $z$ direction; boundary conditions $T=1$ at $z=0, T=0$ at $z=1$ were imposed. The Rayleigh number $\mathbf{R a}$, the dimensionless measure of temperature difference across the layer, was taken to be $\mathrm{Ra}=2000$, close to $\mathrm{Ra}_{c}$ but large enough that the influence of the side walls is expected to be restricted to a region one or two roll wavelengths distant. ${ }^{6}$ At all walls $\mathbf{u}=\mathbf{0}$.

The FLOW $3 D^{7}$ is a code developed to study heat transfer problems subject to realistic, no-slip velocity boundary conditions and a variety of thermal boundary conditions. Upwinding gives FLOW3D good nonlinear stability characteristics: upwinding schemes often also remain qualitatively accurate at parameter values where formally more accurate schemes are unstable or produce meaningless wiggles as a result of Gibbs' phenomenon. The nonlinear equations (1) are solved by first linearizing, solving the resulting equations by standard spaise matrix techniques, and then coupling their solutions as described by Issa. ${ }^{8}$ FLOw3D is fast because it includes sparse matrix solvers that have been carefully tailored for execution on CRAY-architecture machines. Hence the code was chosen to perform direct simulations of the onset of turbulence in convection in shallow tanks, which is ongoing work; however, it is also possible to use FLOw3D efficiently for 2-D problems and we discuss here only results where variation in one horizontal direction, the $y$ direction, was suppressed.

The specification of the 2-D problem (1) is completed by selecting the aspect ration of the box to be 16 , a number not so large that visualizing the whole volume accurately becomes too difficult, and making the side walls nonconducting, i.e., $\partial T / \partial x=0$ at $x=0$ and $x=16$. A $8 \times 98$ mesh was used: at $\mathrm{Ra}=2000$ the Reynolds number $\mathrm{Re}$ of the flow is $O(1)$, so despite the low formal accuracy of the upwind scheme, results may be expected to be quantitatively as well as qualitatively accurate. This was confirmed by repeating some calculations on a $18 \times 194$ mesh.

The initial conditions used were $\mathbf{u}=\mathbf{0}$ everywhere:

$$
T=1-z+\frac{1}{20}\left[1+\cos \left(\pi x n_{0} / 16\right)\right] \text {. }
$$

Thus $n_{0}$ corresponds to the number $n$ of rolls expected to be seen in the resulting convection. To compare with the stability boundary calculated in Ref. 2, separate calculations were begun with $n_{0}=12,13,14, \ldots, 20$, and run to $t=12$, i.e., for about 12 roll turnover times, since $\operatorname{Re}=O(1)$. Solutions with $n=12$ and $n=20$ are not expected because the corresponding wavenumber lies outside the band that is Eckhaus stable. ${ }^{2}$ The other instabilities which limit the band of stable wavenumbers $\alpha$ are three dimensional in character and will not of course be operative in these 2-D simulations; in any case at this Ra, Eckhaus (2-D) instability is the major factor limiting the band.

Simulations confirm that $n=12,19$, and 20 solutions are so unstable that they cannot be said to develop; the outcome of these calculations were flows with, respectively, 14, 17 , and 18 rolls filling the box. There were no surprises from using the other initial conditions: this series of runs indicated that patterns with between 14 and 18 rolls were stable. The corresponding wavenumbers fit closely to the range predicted to be stable in Ref. 2.

However $t=12$ is only a fraction of the horizontal thermal diffusion time scale $=16^{2}$ or 256 in these units; moreover it was noticed that slight changes - in the fourth digitof monitored quantities were still occurring up to this time. Runs were therefore continued with the result shown in Fig. 1. After a long time, which we identify with the horizontal thermal diffusion time scale, the 18-roll solution loses stability. The most significant changes occur near the side walls, and almost simultaneously around $t=27-42$. The roll nearest either wall becomes compressed laterally and the flow close to the wall very slow: in the near-wall region this roll of short wavelength shrinks and gets absorbed by its neighbor (which expands). Therefore a slow readjustment takes place until all rolls have almost the same width. The transition from a 13 - to a 15 -roll solution occurs similarly but in reverse, i.e., either roll near a side wall elongates and a new short wavelength roll grows near the wall. The mechanism, which also eliminates 14-roll solutions (they go to $n=16$ ), is somewhat reminiscent of the way in which the most linearly unstable roll pattern changes as aspect ratio varies, ${ }^{5}$ notably in the way symmetry is preserved. ${ }^{9}$ Finally we remark that the patterns with $n=15,16$, and 17 were found to remain stable for $t$ up to 360 , and no changes in
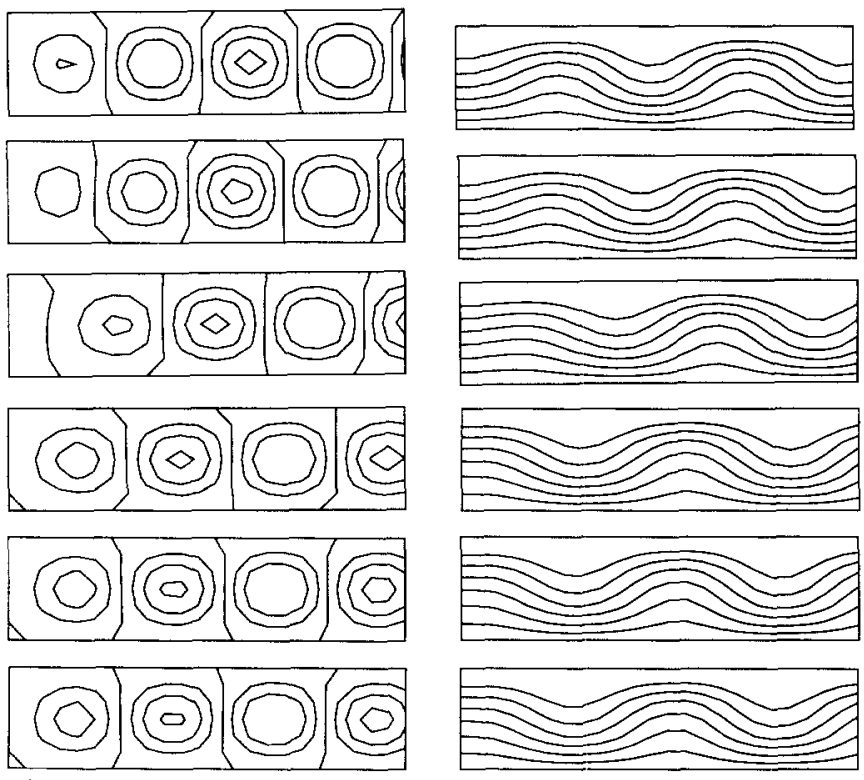

(a)
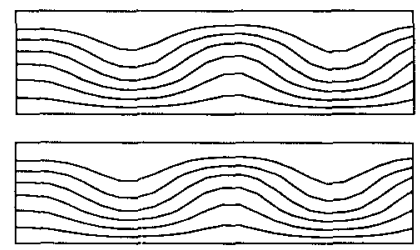

(b)

FIG. 1. (a) Streamlines and (b) isotherms for convection in a $1 \times 16$ box at $R=2000, \sigma=2.5$ as time increases in units of 12 down the page. The region $x \leqslant 4$ is shown; the overall transition is from a pattern with 18 rolls filling the box to one with 16 . 

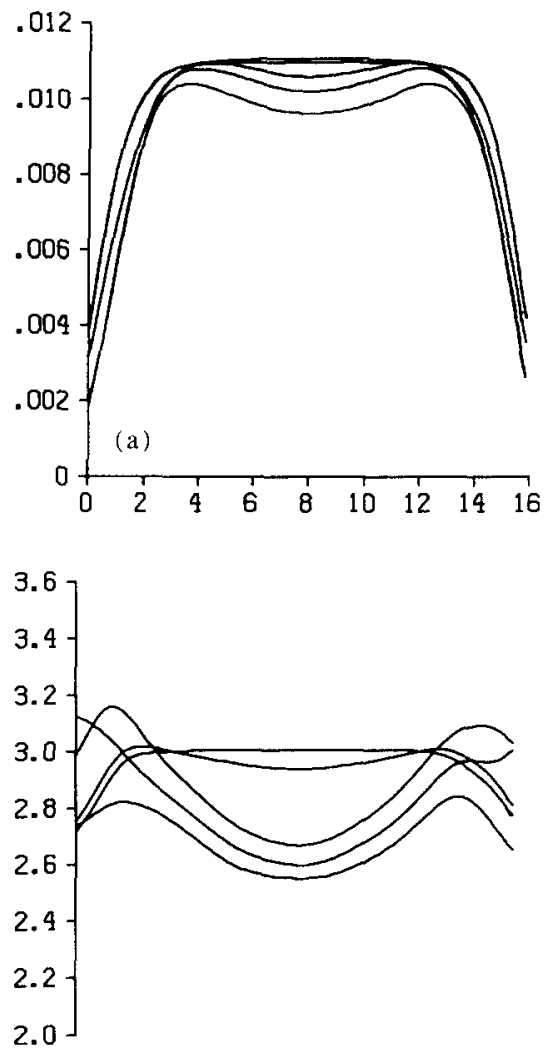

(b)

\begin{tabular}{lllllllll}
\hline 0 & 2 & 4 & 6 & 8 & 10 & 12 & 19 & 16
\end{tabular}

FIG. 2. (a) Amplitude function $A(x)$ and (b) local wavenumber function $k(x)$ for the transition from a 13-roll to a 15-roll pattern. As time increases, $A$ grows while $k$ increases towards more uniform distribution (the plots are for times $t=2.4,4.2,6.0,21$, and 96).

monitored quantities were seen during the last $25 \%$ or more of this interval.

The band of stable wavenumbers is apparently reduced by the long time scale instability from 1.2 to 0.4 , a factor of 3 , at $\mathrm{Ra}=2000$. Runs at other $\mathrm{Ra}$, however, suggest the band is narrowed only by an absolute amount $\Delta \alpha \simeq 0.4$ at either end, rather than by some fixed proportion: the band of stable wavenumbers is narrower the smaller $\mathrm{Ra}$ is. There is no reason why the instability mechanism discovered here should not operate at other Pr. The principal result of this investigation is therefore to answer the old question ${ }^{10}$ about the effect of large but finite box width in favor of those believing that the range of stable $\alpha$ is reduced. ${ }^{4}$ However, the discrete size of the box means that the stability boundary in ( $\mathrm{Ra}, k$ ) space is not two straight lines, ${ }^{4}$ but rather has a stepped appearance.

A qualitative interpretation of the instability in terms of the phase dynamics for convection can be given. Modulation theory methods suggest that the dynamics of convection near threshold are governed by the Newell-WhiteheadSegel equations, "

$$
A_{T}=A+A_{X X}-A|A|^{2}+O\left(\epsilon^{1 / 2}\right),
$$

where $\epsilon=\left(\mathrm{Ra}-\mathrm{Ra}_{c}\right) / \mathrm{Ra}_{c}$ is the reduced Rayleigh number. The amplitude $A$ is related to the horizontal velocity $u$ by

$$
u=\epsilon^{1 / 2} A(X, T) \exp \left(i k_{c} x\right) F(z)+\text { c.c. }+O(\epsilon),
$$

where $F(z)$ describes the vertical structure of the convection roll and $X=\epsilon^{1 / 2} x, T=\epsilon t$ are slow length and time scales, respectively. If the change of variables $A=R e^{i \theta}$ is made, and the flow assumed steady, the equations can be integrated to give

$$
R^{2} K=h, \quad R_{X}^{2}+h^{2} / R^{2}+R^{2}-\frac{1}{2} R^{4}=E,
$$

where $K=\theta_{X}$. It has been argued ${ }^{4}$ that when the width of the box is $L \gg d \epsilon^{-1 / 2}$, the amount of phase winding in the box is limited by the minimum value of $R$, achieved near the boundaries. Cross et al. $^{4}$ have shown that this value is of order $\epsilon^{1 / 2}$ and therefore $h$ is of order $\epsilon^{1 / 2}$ and $K$ of order $\epsilon^{1 / 2}$. Thus the wavenumber $k(x)=k_{c}+\epsilon^{1 / 2} K$ is restricted to be within order $\epsilon$ of the critical value, as opposed to a band of width $\epsilon^{1 / 2}$ in the case of an infinite layer.

For the FLOW3D simulations, $A(x)$ and $k(x)$ were determined by a frequency shift and filtering technique. (The results were found to correlate well with naive measures based on using zeros of $u_{x}$ or $u_{z}$ to determine cell width, then calculating amplitude by integrating $u_{x}^{2}$ or $u_{z}^{2}$ over each cell.) In the evolution studied here (Fig. 2) $k$ is seen first to relax to a nearly constant solution in the interior. Eventually, this relaxation results in an instability in the side wall region. An extra roll is created at the boundary, and the interior layer adjusts to a slightly decreased wavenumber. It is this side wall instability that leads to a reduced band of stable wavenumbers; in sharp contrast to an Eckhaus-type instability which would decrease the wavenumber by introducing a roll pair into the interior of the layer.

\section{ACKNOWLEDGMENTS}

We are most grateful to I. P. Jones of UKAEA/Harwell Laboratory for making available a version of the FLOW3D code.

We are also grateful for the support under AFOSR Contract No. F4962086C0130, NSF Grant No. DMS843187, and ONR Contract No. N001484K0420.

\footnotetext{
a) Permanent address: Culham Laboratory, Abingdon, Oxon OX14 3DB, England.
}

${ }^{1}$ F. H. Busse, Rep. Prog. Phys. 41, 1929 (1978)

${ }^{2}$ E. W. Bolton, F. H. Busse, and R. M. Clever, J. Fluid Mech. 164, 469 (1986).

${ }^{3}$ P. Hall and J. C. Walton, Proc. R. Soc. London Ser. A 358, 199 (1977).

${ }^{4}$ M. C. Cross, P. G. Daniels, P. C. Hohenberg, and E. D. Siggia, J. Fluid Mech. 127, 155 (1983).

${ }^{5}$ I. Catton, Int. J. Heat Mass Transfer 15, 665 ( 1972).

${ }^{6}$ P. Berge and M. Dubois, Contemp. Phys. 25, 535 (1984).

${ }^{7}$ I. P. Jones, J. R. Kightley, C. P. Thompson, and N. S. Wilkes (private communication). FLOw3D has been validated on many convection problems, both by the Harwell group and the present authors, who can provide details on request.

${ }^{8}$ R. I. Issa, J. Comput. Phys. 62, 40 ( 1985 ).

${ }^{9}$ K. A. Cliffe and K. H. Winters, submitted to J. Comput. Phys.

${ }^{10}$ F. H. Busse, J. Fluid Mech. 30, 625 ( 1967).

${ }^{11}$ A. C. Newell and J. A. Whitehead, J. Fluid Mech. 38, 279 (1969); M. C Cross and A. C. Newell, Physica D 10, 299 (1984). 